\title{
Minimizing Flow Completion Times in Data Centers
}

\author{
Ali Munir, Ihsan A. Qazi, Zartash A. Uzmi, Aisha Mushtaq, Saad N. Ismail, M. Safdar Iqbal, Basma Khan \\ Computer Science Department, LUMS \\ Email: \{ali.munir,ihsan.qazi,zartash,14100142,14100055,12100005,basma.khan\}@lums.edu.pk
}

\begin{abstract}
For provisioning large-scale online applications such as web search, social networks and advertisement systems, data centers face extreme challenges in providing low latency for short flows (that result from end-user actions) and high throughput for background flows (that are needed to maintain data consistency and structure across massively distributed systems). We propose $L^{2}$ DCT, a practical data center transport protocol that targets a reduction in flow completion times for short flows by approximating the Least Attained Service (LAS) scheduling discipline, without requiring any changes in application software or router hardware, and without adversely affecting the long flows. $\mathrm{L}^{2} \mathrm{DCT}$ can co-exist with TCP and works by adapting flow rates to the extent of network congestion inferred via Explicit Congestion Notification (ECN) marking, a feature widely supported by the installed router base. Though $\mathrm{L}^{2} \mathrm{DCT}$ is deadline unaware, our results indicate that, for typical data center traffic patterns and deadlines and over a wide range of traffic load, its deadline miss rate is consistently smaller compared to existing deadlinedriven data center transport protocols. $L^{2}$ DCT reduces the mean flow completion time by up to $50 \%$ over DCTCP and by up to 95\% over TCP. In addition, it reduces the completion for 99th percentile flows by $37 \%$ over DCTCP. We present the design and analysis of $\mathrm{L}^{2} \mathrm{DCT}$, evaluate its performance, and discuss an implementation built upon standard Linux protocol stack.
\end{abstract}

\section{INTRODUCTION}

Data centers are now being used as a critical infrastructure for high-revenue online services such as web search, social networking, advertisement systems, and recommendation systems. Such data center applications pose demanding latency requirements and even a small fraction of a second can make a quantifiable difference in user experience thus impacting the revenue. For example, Google observed a $20 \%$ traffic reduction from an extra $500 \mathrm{~ms}$ of latency (introduced inadvertently), and Amazon found that every additional $100 \mathrm{~ms}$ of latency costs them a $1 \%$ loss in business revenue [1], [2].

Large-scale online applications are typically hosted at data centers and follow the Partition/Aggregate workflow pattern, in which user requests are partitioned amongst layers of worker nodes within the data center and the results from the workers are then combined by an aggregator node before a final response is sent back to the user [3], [4].

Provisioning these applications leads to data center traffic that is a mix of short and long flows. Prior work shows that most flows are short arising from end-user actions, however most bytes are contained in a very small number of background long flows that are needed to maintain data consistency and structure across massively distributed systems [3]. The timeliness of response to the end-user is determined by the short foreground flows while the quality of response is determined by both the short and the long flows. Long-lived TCP flows cause the length of the bottleneck queue to grow until packets get dropped. When long flows and latency-sensitive short flows share the same queue, short flows experience increased latency due to queue buildup by long flows [1], [5], [6].

In this paper, we present $\mathrm{L}^{2} \mathrm{DCT}$ (Low Latency Data Center Transport), a practical data center transport protocol that targets a reduction in the completion times for short flows. $\mathrm{L}^{2} \mathrm{DCT}$ can be deployed incrementally as it can co-exist with TCP and does not require any changes to router hardware or application software. At the heart of $\mathrm{L}^{2} \mathrm{DCT}$ is the additive increase and backoff mechanism for setting the transport layer window size. Under this mechanism, end hosts make use of the information inferred from Explicit Congestion Notification $(\mathrm{ECN})$ marking and adjust their flow rates (by setting the window size) based on the amount of data a flow has already sent. Intuitively, conservative backoff and aggressive increase for short flows allows these flows to finish relatively quickly.

A number of transport protocols have previously been proposed for large-scale data center applications with Partition/Aggregate workflow.

One class of data center protocols approximate the processor sharing (PS) discipline by dividing the link bandwidth equally among flows [1], [7], [8]. This solution ignores the disparate requirements for short foreground and long background flows. Furthermore, it has previously been shown that although the PS discipline leads to fairness, it is far from optimal in terms of minimizing the average flow completion time (AFCT) [9].

Another class of data center protocols assign deadlines to flows and try to meet those deadlines as the main objective [4], [6]. Such protocols require changes to applications (for passing deadline and/or flow size information) and may need router hardware modifications [4]. Furthermore, there is no established basis for accurately choosing the deadlines, which are currently set based on user experience surveys [1], [4].

$\mathrm{L}^{2} \mathrm{DCT}$ focuses on reducing the completion times for short flows. PDQ [10] shares a similar objective and improves the flow completion times over TCP, RCP [8] and $\mathrm{D}^{3}$ [4]. However, it requires modifications to switch hardware and software and is incompatible with TCP, leading to practical difficulties with deployment. Indeed, a protocol can optimally minimize the AFCT by using the Shortest Remaining Processing Time (SRPT) scheduling discipline. However, SRPT requires knowledge of flow sizes (which may or may not be available), a centralized scheduler, and incurs an overhead for passing flow size information to the scheduler. $\mathrm{L}^{2} \mathrm{DCT}$ overcomes all these limitations: it does not need a centralized scheduler, is compatible with TCP, does not require any software or hardware support from the routers (except for the ECN marking which is a standard feature in present-day routers [1], [6]), and is easy to implement (requires fewer than 75 lines of code change to TCP in the Linux kernel). 


\begin{tabular}{|c|c|c|}
\hline Flow ID & Size & Start Time \\
\hline A & 1 & 1 \\
\hline B & 2 & 2 \\
\hline C & 4 & 0 \\
\hline
\end{tabular}

(a)

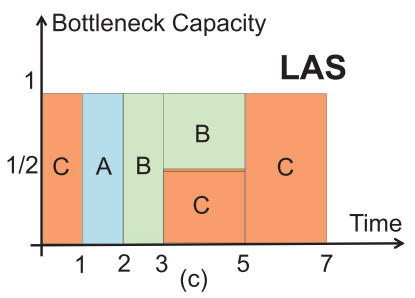

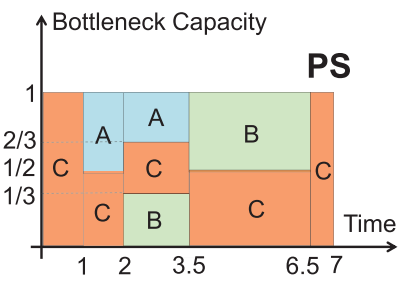

(b)

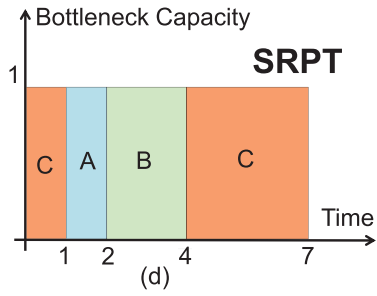

Fig. 1. Motivating example.

Practicality of $\mathrm{L}^{2} \mathrm{DCT}$ also stems from the fact that it approximates the Least Attained Service (LAS) scheduling discipline, which does not require flow size information. LAS also targets a reduction in AFCT and closely approximates SRPT [11] even though it is not optimal, as illustrated in Figure 1. Three flows (A, B, and C), having different transfer sizes, arrive at different times. Assuming a fluid traffic model i.e., with infinitesimally small units of transmission, the progress of flows with fair sharing, SRPT, and LAS is as shown. With fair sharing or Processor Sharing (PS), the flows A, B, and $\mathrm{C}$ finish at times 3.5, 6.5, and 7, respectively and have an AFCT of $\frac{2.5+4.5+7}{3}=4.67$. With SRPT, the AFCT becomes $\frac{1+2+7}{3}=3.33$ and with LAS, the AFCT is $\frac{1+3+7}{3}=3.67$.

The congestion control mechanism of $\mathrm{L}^{2} \mathrm{DCT}$ approximates LAS as follows: in the face of congestion, long flows back off aggressively while short flows do so conservatively. In contrast, the additive increase of congestion window is more aggressive in case of short flows. This intuitively favors the short flows to finish quickly without causing any starvation of long flows. Furthermore, window size adjustment based on the extent of congestion, as estimated by ECN marking, allows long flows to achieve high throughput in the absence of short flows and prevents congestion collapse.

$\mathrm{L}^{2}$ DCT reduces the AFCT by up to $50 \%$ over DCTCP and by up to $95 \%$ over TCP. It also reduces the completion time for the 99th percentile flows by $37 \%$ over DCTCP. Though $\mathrm{L}^{2} \mathrm{DCT}$ focuses on AFCT and is deadline unaware, our results also show that it would miss fewer deadlines compared to existing deadline-driven data center transport protocols, for typical data center traffic patterns and deadlines and over a wide range of traffic load. Altogether, this paper makes the following contributions:

- A data center transport protocol $\mathrm{L}^{2} \mathrm{DCT}$ that targets a reduction in flow completion times, is incrementally deployable, requires no changes to the data center application code base or the router hardware, and is able to co-exist with TCP.

- Design and analysis of the congestion control mechanism used in $\mathrm{L}^{2} \mathrm{DCT}$.

- Extensive evaluation of $\mathrm{L}^{2} \mathrm{DCT}$ (using at-scale simulations) in comparison with TCP and other proposed data center transport protocols under typical data center traffic patterns for large-scale online applications over a wide range of workloads, measuring the impact in: average flow completion times, proportion of deadlines missed, per flow throughput in a multihop setting, and bottleneck queue length.

- A small-scale testbed evaluation using an implementation built upon the standard Linux protocol stack.

The rest of the paper is organized as follows. We describe the details of $\mathrm{L}^{2} \mathrm{DCT}$ and present its analysis in Section II. We evaluate L $^{2}$ DCT's performance in Section III. Linux implementation and real testbed results are presented in Section IV. We discuss related work in Section V, followed by some discussion and future work in Section VI. We offer concluding remarks in Section VII.

\section{L $^{2}$ DCT PROTOCOL}

$\mathrm{L}^{2} \mathrm{DCT}$ modulates the congestion window size based on estimated flow sizes as well as the extent of congestion in the network. Flow sizes are estimated based on the amount of data a flow has sent so far. This enables $\mathrm{L}^{2} \mathrm{DCT}$ to adapt congestion window sizes in a size-aware manner without requiring flow size information and approximate scheduling disciplines such as LAS. With such size-aware congestion management, $\mathrm{L}^{2} \mathrm{DCT}$ is able to significantly reduce the AFCT.

\section{A. Congestion Avoidance Algorithm}

The congestion avoidance algorithm used by $\mathrm{L}^{2} \mathrm{DCT}$ has two components, one at the sender side and the other at the routers. Like DCTCP [1], a router marks all packets by setting the Congestion Experienced (CE) bits using ECN [12] when the queue length exceeds a certain threshold. $\mathrm{L}^{2} \mathrm{DCT}$ senders measure the extent of network congestion by maintaining a weighted average of the fraction of marked packets, $\alpha$, as:

$$
\alpha=g \times F+(1-g) \times \alpha
$$

where $F$ is the fraction of packets marked in the most recent window, and $g$ is the weight given to new samples.

In order to realize different scheduling disciplines, such as SRPT and LAS, which prioritize flows based on their sizes, $\mathrm{L}^{2} \mathrm{DCT}$ modulates the congestion window size of each flow based on $\alpha$ and a weight. In the context of LAS, these weights are assigned based on the amount of flow data sent so far but can differ across scheduling disciplines (which we discuss in Section III-E). These weights implicitly define priorities of a flow. Based on these weights and $\alpha$, we determine $k$, the increase in congestion window per round-trip time (RTT) and the backoff penalty $b$, as follows:

$$
\begin{gathered}
k=w_{c} / w_{\max } \\
b=\alpha^{w_{c}}
\end{gathered}
$$

where $w_{c} \in\left[w_{\min }, w_{\max }\right]$ is the current flow weight, $w_{\min }$ is the minimum weight, and $w_{\max }$ is the maximum weight any flow can assume. We evaluate the impact of these bounds on $w_{c}$ in Section III. Each flow starts by setting $w_{c}$ to $w_{\max }$. As flows send more data, $w_{c}$ decreases before converging to $w_{\text {min }}$. Observe that since $w_{c} \leq w_{\max }$ and $\alpha \leq 1$, therefore 


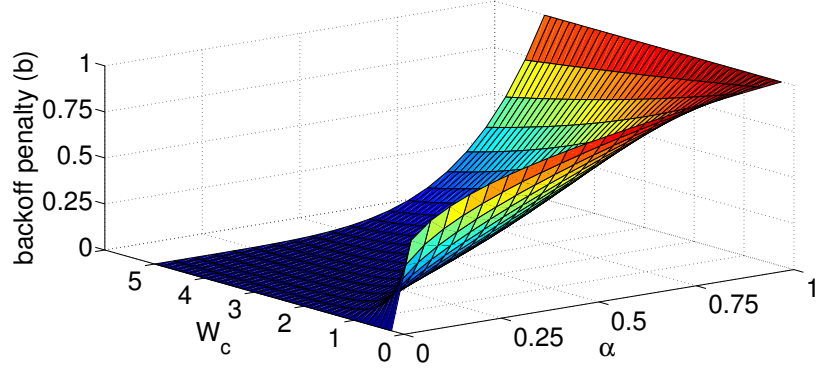

Fig. 2. Changes in backoff penalty $b$ as a function of $\alpha$ and $w_{c}$.

$k \leq 1$ and $b \leq 1$, respectively. While the increase in window size per RTT can be more than one but due to burstiness in packet arrivals in data center applications [5], we limit $k$ to at most 1, which is the increase factor used by TCP [1].

When a marked ACK (i.e., with ECN-Echo flag set) is received, $\mathrm{L}^{2} \mathrm{DCT}$ uses $b$ to reduce the window size as:

$$
\text { cwnd }=\text { cwnd } \times(1-b / 2) \text {. }
$$

Note that TCP, unlike $\mathrm{L}^{2}$ DCT, always cuts its window size by half $^{1}$. When no packets are CE-marked, the window size is increased as:

$$
\text { cwnd }=\text { cwnd }+k \text {. }
$$

Therefore, when congestion is high $(\alpha=1$ and $b=1)$ the window size is reduced by half, similar to TCP. When $\alpha=0$, and so is $b$, window increase depends on $w_{c}$. When $\alpha$ varies between 0 and 1 , the window size is adapted based on $k$ and $b$. In particular, flows with high weight (i.e., short flows) incur a smaller backoff penalty and apply a larger $k$ compared to flows with smaller weights (i.e., long flows). Note that when $w_{c}=w_{\max }$, the window size is increased by one packet, similar to TCP.

Flow weights: The weight $w_{c}$ decreases with the amount of data a flow has already sent. Some possible functions for $w_{c}$ include $1 / s$ and $e^{-s}$, where $s$ is the data sent so far. We discuss the choice of the weight function in Section III.

\section{B. Understanding Congestion Behavior}

The congestion behavior of $\mathrm{L}^{2} \mathrm{DCT}$ depends on $w_{c}$ and $\alpha$ as they impact the window increase and decrease policies. We now discuss how the choice of $\mathrm{L}^{2}$ DCT's window control policies allows it to achieve the following goals:

1) When short flows and long flows co-exist, the latter should relinquish bandwidth to allow the former a greater short term share of the bandwidth.

2) When only long flows are present, they should be able to achieve high throughput and not be penalized any more than regular TCP or DCTCP.

3) When congestion becomes severe (i.e., $\alpha$ is close to 1), all flows should converge to applying full backoff, similar to TCP, to prevent congestion collapse.

The first goal suggests that short flows should increase their window faster than long flows and backoff less. Consequently, we vary $k$ as a function of $w_{c}$. When a new flow starts, it

\footnotetext{
${ }^{1} \mathrm{TCP}$ and $\mathrm{L}^{2} \mathrm{DCT}$ both reduce their window size at most once per RTT.
}

increases its window by 1 pkt/RTT (similar to TCP). However, as the flow transmits more packets, its weight decreases, leading to a proportional decrease in the increase/RTT. This helps in prioritizing short flows over long flows.

Figure 2 shows the backoff penalty as a function of $w_{c}$ and $\alpha$. Observe that when $0<w_{c}<1$ (i.e., long flows), $b$ increases rapidly even with small increases in $\alpha$, and approaches 1 as $\alpha$ tends to 1 . This implies that minor congestion causes rapid reduction in the window sizes of long flows but severe congestion (e.g. $\alpha=1$ ) does not penalize such flows any more than regular TCP or DCTCP as suggested by the second goal.

When $w_{c}>1$ (i.e., short flows), $b$ increases slowly in response to increases in $\alpha$, until $\alpha$ approaches 1 , at which point $b$ rapidly converges to 1 . Therefore, minor congestion does not penalize short flows by much, which allows such flows a greater short-term share of the bandwidth to finish quickly. However, severe congestion causes a full backoff.

When congestion continues to grow in severity after long flows have backed off, then two scenarios are possible: $(i)$ there are many short flows, who are not reducing their share of the bandwidth (ii) there may be TCP flows who are consuming bandwidth. Both these situations are handled because as $\alpha$ tends to 1 , even short flows will throttle themselves, thus allowing TCP and other short flows to make progress. Consequently, the shortest flows will have the largest share of the bandwidth.

\section{Analysis}

To understand the impact of $w_{c}$ and $k$ on the steady state behavior of $\mathrm{L}^{2} \mathrm{DCT}$, we now present the analysis of $\mathrm{L}^{2} \mathrm{DCT}$ in a simplified setting. We consider $N$ long-lived flows with identical RTTs $T$ and weight $w_{c}$, sharing a single bottleneck link of capacity $C$. It is further assumed that the $N$ flows are synchronized i.e., their window dynamics (or sawtooths) are in-phase. Of course, this assumption is only realistic when $N$ is small, however, this is the case we care about most in data centers, where responses from worker nodes are synchronized [7]. We further assume that $w_{c}$ is fixed. In reality, $w_{c}$ changes over time, however, this assumption still allows us to capture the impact of $w_{c}$ on the protocol performance.

Due to flow synchronization, the window sizes of $N$ flows follow identical sawtooths, and therefore, the queue size process also follows a sawtooth [13]. We are interested in determining the backoff penalty $b$ as a function of $w_{c}, k$, and the maximum window size $\left(W^{o}\right)$ as well as quantities which completely specify the queue sawtooth: the amplitude of queue oscillations $(A)$, period of oscillations $\left(T_{C}\right)$, and the maximum queue length $\left(Q_{\max }\right)$.

With synchronized flows, the queue length exceeds the marking threshold $K$ for exactly one RTT in each period of the sawtooth, before the sources receive ECN marks and reduce their window sizes accordingly. Therefore, we compute the fraction of marked packets, $\alpha$, by dividing the number of ACKs received during the last RTT by the total number of ACKs received during the full period of the sawtooth, $T_{C}$.

We now consider one of the flows and determine its backoff penalty. Let $X\left(W_{1}, W_{2}\right)$ denote the number of packets sent by 
a flow, while its window increases from $W_{1}$ to $W_{2}>W_{1}$. This takes $\left(W_{2}-W_{1}\right) / k$ RTTs $^{2}$ during which the average window size is $\left(W_{1}+W_{2}\right) / 2$.

$$
X\left(W_{1}, W_{2}\right)=\left(W_{2}^{2}-W_{1}^{2}\right) / 2 k
$$

Let $W^{o}=(C T+K) / N$. This is the window size at which the queue length reaches $K$, and switch starts marking the packets with the CE codepoint. During the round-trip time it takes for the sender to react to these marks, another $W^{o}$ packets have been sent. Hence, fraction of marked packets, $\alpha$, can be calculated by,

$$
\begin{aligned}
& \alpha=X\left(W^{o}, W^{o}+k\right) / X\left(\left(W^{o}+k\right)(1-b / 2), W^{o}+k\right) \\
= & \left(\left(W^{o}+k\right)^{2}-\left(W^{o}\right)^{2}\right) /\left(\left(W^{o}+k\right)^{2}-\left(W^{o}+k\right)^{2}(1-b / 2)^{2}\right)
\end{aligned}
$$

Simplifying and rearranging the equation gives,

$$
\alpha\left(b-b^{2} / 4\right)=\left(2 W^{o} k+k^{2}\right) /\left(W^{o}+k\right)^{2}
$$

Assuming $b$ is small, we can rewrite the equation as,

$$
\alpha b=\left(2 W^{o} k+k^{2}\right) /\left(W^{o}+k\right)^{2}
$$

Plugging the value of $b=\alpha^{w_{c}}$, gives us,

$$
\begin{gathered}
\alpha=\left[\left(2 W^{o} k+k^{2}\right) /\left(W^{o}+k\right)^{2}\right]^{1 /\left(w_{c}+1\right)} \\
\text { and } b=\left[\left(2 W^{o} k+k^{2}\right) /\left(W^{o}+k\right)^{2}\right]^{w_{c} /\left(w_{c}+1\right)}
\end{gathered}
$$

Note that when $w_{c}=1$ and $k=1$, we obtain $\alpha$ for DCTCP [1]. The amplitude of oscillation in the window size of a single flow, $D$, is given by,

$$
D=\left(W^{o}+k\right)-\left(W^{o}+k\right)(1-b / 2)=b\left(W^{o}+k\right) / 2
$$

As there are $N$ flows in total, $A$ can be computed as follows,

$$
A=N D=N b\left(W^{o}+k\right) / 2 \approx \frac{N W^{o}}{2} \cdot\left(\frac{2 k}{W^{o}}\right)^{w_{c} /\left(w_{c}+1\right)}
$$

where the final expression assumes that $W^{o}>>k$. The period of the oscillations and the maximum queue length are,

$$
\begin{gathered}
T_{c}=D=b\left(W^{o}+k\right) / 2 \\
Q_{\max }=N\left(W^{o}+k\right)-C \times T=K+N k
\end{gathered}
$$

We compared the accuracy of the above results with NS2 simulations. Figure 3 shows the results for $w_{c} \in\{0.5,1\}$ and $N \in\{1,2\}$ on a $1 \mathrm{Gbps}$ link with a RTT of $300 \mu \mathrm{s}$. Observe that the analysis provides a fairly accurate prediction of the window dynamics when $w_{c}=1$. For $w_{c}=0.5$, the analysis yields larger variations in window size compared to simulations due to the continuous approximation we made. An important property, revealed by Equation 9, is that the amplitude of the queue oscillations of $\mathrm{L}^{2} \mathrm{DCT}$ is in $O\left((C \times T)^{1 /\left(w_{c}+1\right)}\right)$ when $N$ is small. In particular, the queue oscillations become independent of $C \times T$ (i.e., the BDP) when $w_{c}$ is large and the ratio $1 /\left(w_{c}+1\right)$ approaches zero

\footnotetext{
${ }^{2}$ Note that when $k<1$, a flow sends $W$ packets/RTT until the window becomes $W+1$. However, we found the above continuous approximation to be fairly accurate compared to the precise expressions, which require solving $\alpha$ numerically.
}

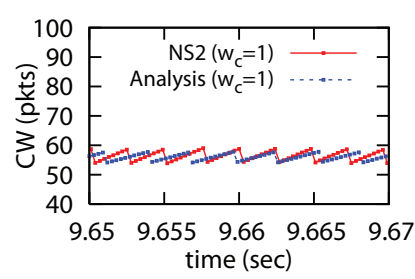

(a) $N=1, w_{c}=1$

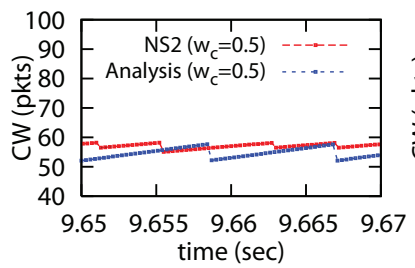

(c) $N=1, w_{c}=0.5$

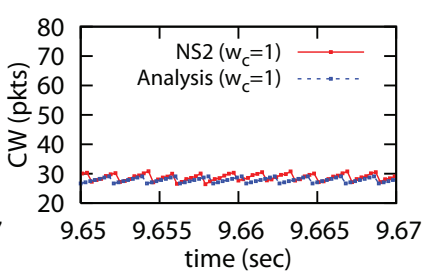

(b) $N=2, w_{c}=1$

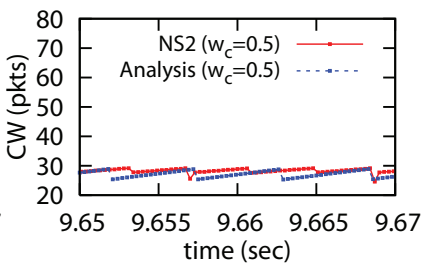

(d) $N=2, w_{c}=0.5$
Fig. 3. Comparison of the window size dynamics predicted by the analysis with NS2 simulations for different weights $w_{c}$.

because senders backoff less. When $w_{c}=1$ and $N$ is small, the amplitude is in $O(\sqrt{C \times T})$, which is also the case for DCTCP [1]. Finally, when $w_{c}$ approaches zero, the oscillations become similar to those of TCP. The lower bound we chose on $w_{c}$ (i.e., $w_{\min }=0.125$ ) implies that the amplitude of the queue oscillations for small $N$ in the worst case is in $O\left((C \times T)^{8 / 9}\right)$, which is much smaller than that of TCP. This implies that we can have a small marking threshold $K$ without losing throughput in the low statistical multiplexing regime seen in data center environments. We know that,

$$
Q_{\min }=Q_{\max }-A
$$

To determine the lower bound on $K$, we minimize (12) over $N$ to get,

$$
K>\frac{w_{c}^{w_{c}}(C \times T)}{2\left(w_{c}+1\right)^{w_{c}+1}-w_{c}^{w_{c}}}
$$

In the worst-case scenario, where $w_{c}=0.125$, we get $K>$ $(C \times T) / 2$.

\section{EVALUATION}

We evaluate the performance of $\mathrm{L}^{2} \mathrm{DCT}$ using at-scale simulations and a real Linux implementation. First, we evaluate $\mathrm{L}^{2} \mathrm{DCT}$ using a benchmark generated from the traffic measurement study conducted in [1]. Second, we evaluate $\mathrm{L}^{2}$ DCT's throughput and queuing behavior in single and multihop environments. Finally, we evaluate $\mathrm{L}^{2} \mathrm{DCT}$ 's performance when it co-exists with TCP.

We compare the performance of $\mathrm{L}^{2} \mathrm{DCT}$ with DCTCP and TCP SACK with drop-tail queueing. For deadline-aware scenarios, we compare $\mathrm{L}^{2} \mathrm{DCT}$ with $\mathrm{D}^{2} \mathrm{TCP}$ [6], a recently proposed deadline-aware transport protocol. Unless otherwise stated, we use a single-rooted tree; a commonly used data center topology for our evaluation [1], [4], [7], [14]. In our simulations, we use $1 \mathrm{Gbps}$ interfaces, round-trip propagation delay of $300 \mu \mathrm{s}$, and a static buffer size of 250 packets unless stated otherwise. We set the $\mathrm{RTO}_{\min }$ of all protocols to be $10 \mathrm{~ms}$ as suggested by previous studies [1], [14]. We set the parameters of DCTCP and $\mathrm{L}^{2}$ DCT to match those in [1]. In 


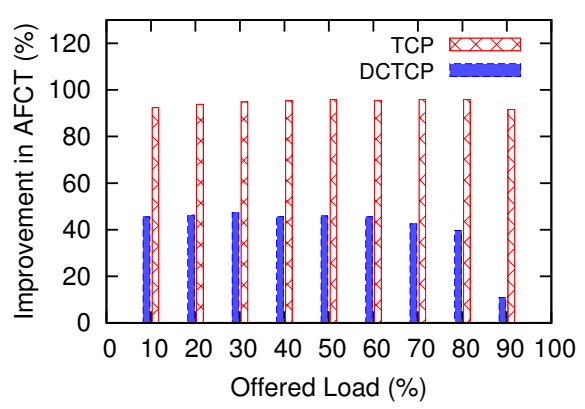

(a)

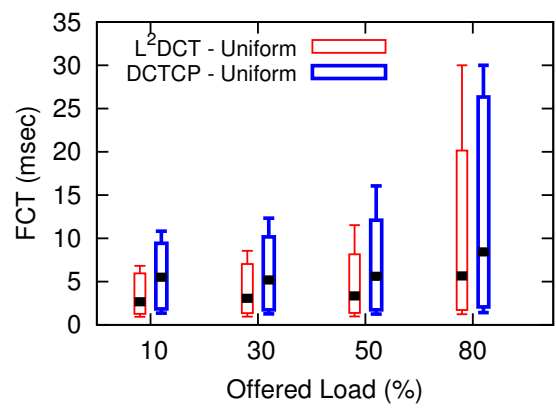

(b)

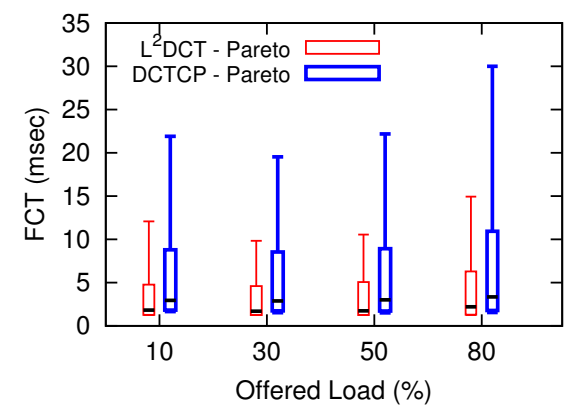

(c)

Fig. 4. Benchmark results at varying traffic loads. (a) Improvement in AFCT of $\mathrm{L}^{2} \mathrm{DCT}$ over DCTCP and TCP. $1^{\text {st }}, 5^{\text {th }}, 50^{\text {th }}$, $95^{\text {th }}$, and $99^{\text {th }}$ percentile completion times of $\mathrm{L}^{2}$ DCT and DCTCP with two long-lived flows along with (b) uniformly distributed flow sizes and (c) Pareto distributed flow sizes.

particular, the weighted averaging factor $g$ is set to $1 / 16$ and the marking threshold $K$ to 20 . For $\mathrm{L}^{2} \mathrm{DCT}$, we cap $w_{c}$ between 2.5 and 0.125 , except in cases where we explore the effect of varying the cap on $w_{c}$. In our evaluation, we assume that $w_{c}$, which is initially 2.5 , stays constant when the amount of data sent so far is $<200 \mathrm{~KB}$, and then decreases linearly to 0.125 for $1 \mathrm{MB}$, and stays constant afterwards. This matches the traffic profiles observed in real data centers, where delaysensitive traffic is generally less than $200 \mathrm{~KB}$ and long-lived flows are of $>1 \mathrm{MB}$ size [1], [5].

\section{A. Data Center Specific Impairments}

1) Benchmark Settings: We generate short query traffic with flow sizes drawn from the interval [2 KB, $98 \mathrm{~KB}]$ using a uniform distribution, as done in a prior study [4]. In addition, we generate two long-lived flows, which represents the $75^{\text {th }}$ percentile traffic multiplexing in data center networks [1].

Figure 4 shows the flows completion time results as a function of the offered traffic load. Observe that $\mathrm{L}^{2} \mathrm{DCT}$ improves the AFCT over DCTCP and TCP by up to $45 \%$ and $95 \%$, respectively (see Figure 4(a)). The improvement in AFCT over DCTCP is at least $40 \%$ for $10-20 \%$ load, which is a realistic load in present-day data centers [4], [5], [6]. L ${ }^{2}$ DCT also improves both the $95^{t h}$ and $99^{\text {th }}$ percentile of completion times by up to $37 \%$ compared to DCTCP (see Figure 4(b)).

Figure 5 shows the corresponding throughput of long background flows. Observe that for data center traffic loads of $10 \%$, there is a difference in the throughput between $\mathrm{L}^{2} \mathrm{DCT}$ and DCTCP of about $6.7 \%$. At higher loads, more short flows arrive per second, which increases this difference. As we discuss in Section III-B.3, throughput of long flows can be increased in these scenarios by a corresponding reduction in flow completion times by adjusting the cap on $w_{c}$.

2) Pareto Distributed Traffic: We generated flows with sizes drawn from a Pareto distribution with mean $50 \mathrm{~KB}$ and shape 1.2. This yields flow sizes that capture realistic data center workloads [1]. In these settings, $\mathrm{L}^{2} \mathrm{DCT}$ improves over DCTCP at all loads as shown in Figure 4(c), with $99^{\text {th }}$ percentile of FCT improved by up to $53 \%$.

3) Incast Behavior: With $\mathrm{L}^{2} \mathrm{DCT}$, short flows behave more aggressively to increase their share of the network bandwidth. Consequently, $\mathrm{L}^{2} \mathrm{DCT}$ may exacerbate Incast. To study the

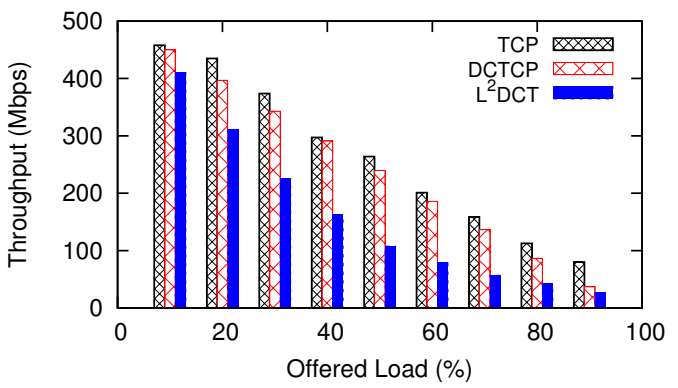

Fig. 5. Throughput of long flows under the benchmark settings.

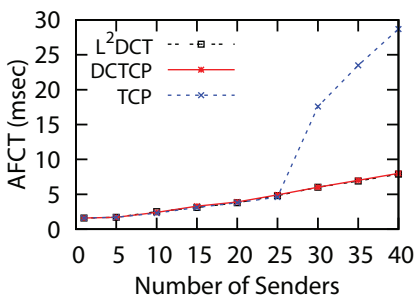

(a)

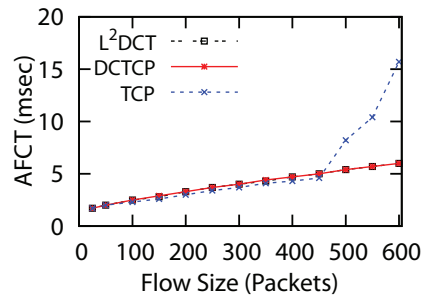

(b)
Fig. 6. AFCT under the Incast scenario. (a) Varying number of senders with Uniform flow sizes with $25 \mathrm{~KB}$ mean. (b) Varying flow size with client requests from 5 different servers.

Incast behavior, we assume a topology where multiple servers send data (responses to queries) to one aggregator simultaneously through a single bottleneck link.

a) Impact of Number of Sending Servers: We vary the number of simultaneous senders from 1 to 40 as done in [1] and flow sizes are generated uniformly at random with a mean of $25 \mathrm{~KB}$. Even though $\mathrm{RTO}_{\min }$ is set to $10 \mathrm{~ms}$, TCP performance still degrades when the number of senders increases beyond 25 . In contrast, even though $\mathrm{L}^{2} \mathrm{DCT}$ uses more aggressive parameters for short flows, its stringent marking policy allows Incast to be mitigated as shown in Figure 6(a).

b) Impact of Flow Size: We assume 5 sending servers and vary the mean flow size (i.e., the size of response from each server) from $50 \mathrm{~KB}$ to $600 \mathrm{~KB}$. As the size of the flow increases, AFCT for $\mathrm{L}^{2} \mathrm{DCT}$ and DCTCP degrades gracefully. However, AFCT with TCP starts degrading when the mean flow size is increased beyond $400 \mathrm{~KB}$ (see Figure 6(b)). 


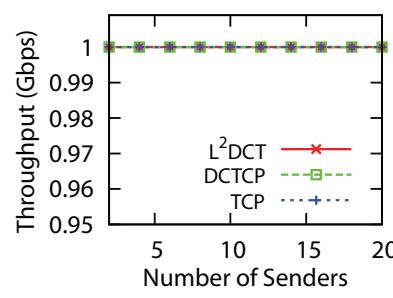

(a)

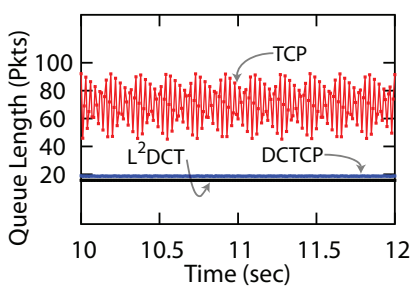

(b)
Fig. 7. Performance of long-lived $\mathrm{L}^{2} \mathrm{DCT}$ flows. (a) Throughput achieved by flows. (b) Queue length dynamics for two long-lived flows.

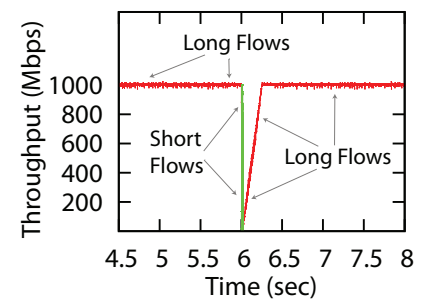

(a)

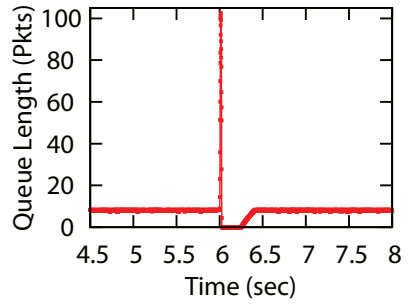

(b)
Fig. 8. Performance of two long $\mathrm{L}^{2} \mathrm{DCT}$ flows when a sudden burst of short flows arrives at time $t=6 \mathrm{~s}$. (a) Throughput (b) Queue length dynamics.

\section{B. $L^{2}$ DCT Specific Testing}

We now evaluate how $\mathrm{L}^{2} \mathrm{DCT}$ performs as a congestion control protocol.

1) Single Bottleneck Scenario: In this scenario, we evaluate the throughput performance of long-lived $\mathrm{L}^{2} \mathrm{DCT}$ flows and perform comparison with TCP and DCTCP. Figure 7(a) shows the throughput of $\mathrm{L}^{2} \mathrm{DCT}$, DCTCP, and TCP flows as a function of the number of senders, each generating a single long-lived flow. Observe that all protocols achieve $~ 100 \%$ throughput. However, $\mathrm{L}^{2} \mathrm{DCT}$ and DCTCP maintain much smaller queue length compared to TCP as shown by Figure 7(b). $\mathrm{L}^{2} \mathrm{DCT}$ is able to maintain small queues due to gentle backoffs based on the extent of network congestion.

2) Effect of Sudden Short Flow Bursts: We now consider the scenario where a sudden burst of short flows arrive while long-lived flows are active. This is a fairly common scenario in data center environments.

Figure 8(a) shows the throughput of flows when 50 short flows, each of size $50 \mathrm{~KB}$, arrive simultaneously in the presence of an ongoing long-lived flow. Observe that $\mathrm{L}^{2} \mathrm{DCT}$ quickly adapts to the sudden bursts of short flows converging to full link utilization afterwards. In particular, the arrival of short flows increases the queue occupancy, causing the long flows to backoff more than short flows (see Figure 8(b)). When short flows complete, long flow quickly grabs the entire bottleneck capacity. Note that the low queue occupancy with $\mathrm{L}^{2} \mathrm{DCT}$ implies that there is more room in the queue for absorbing packet bursts. Moreover, long flows ( $>1 \mathrm{MB}$ ) use $k=0.05$, which means they take $\sim 200 \mathrm{~ms}$ to achieve $1 \mathrm{Gbps}$.

3) Impact of the Weight Function: The performance achieved by $\mathrm{L}^{2} \mathrm{DCT}$ depends on the weights assigned to flows. Extremely low or high values may cause undesirable behavior due to which we cap $w_{c}$ to be within $\left(w_{\max }=2.5\right.$, $\left.w_{\min }=0.125\right)$. In this section, we evaluate the impact of varying $w_{\max }$ and $w_{\min }$.
Figures 9(a) and 9(b) show the impact of varying $w_{\max }$ on the AFCT of short flows and the throughput of long-lived flows when the offered load is $20 \%$. Observe that increasing $w_{\max }$ reduces the AFCT as well as the throughput of long flows. This happens because increasing $w_{\max }$ makes short flows more aggressive, which leads to higher values for $\alpha$. Since long flows backoff significantly even for small $\alpha$, this reduces their throughput. Next, we vary $w_{\min }$. Observe that the AFCT of short flows and the throughput of long flow decreases when $w_{\min }$ is decreased as shown in Figures 9(c) and 9(d). This happens because decreasing $w_{\text {min }}$, increases the backoff factor and lowers the additive increase for long flows, which reduces their throughput in the presence of short flows. Therefore, we set $w_{\max }$ to 2.5 and $w_{\min }$ to 0.125 to achieve a compromise between the performance of short and long flows.

4) Deadline Constrained Flows: We now evaluate the performance of $\mathrm{L}^{2} \mathrm{DCT}$ when flows have deadlines associated with them and compare its performance with $\mathrm{D}^{2} \mathrm{TCP}[6]$, a recently proposed deadline-aware protocol.

We replicate the traffic settings of Section III-A.1, and determine the number of flows missing their deadlines. To generate deadlines, we use the same approach as employed in [4]. In particular, flow deadlines are generated using the exponential distribution with mean $40 \mathrm{msec}$ (tight deadlines), $60 \mathrm{msec}$ (moderate deadlines) and $80 \mathrm{msec}$ (lax deadlines). Figure 10(a), 10(b), and 10(c) show that $\mathrm{L}^{2} \mathrm{DCT}$ outperforms all protocols across a range of traffic loads including $\mathrm{D}^{2} \mathrm{TCP}$, which specifically accounts for flow deadlines. Since deadlines are typically associated with short flows, these results suggest that a deadline agnostic protocol, which minimizes completion times, can achieve better performance than deadline-aware protocols.

\section{Multiple Bottleneck Scenario}

To evaluate L $^{2}$ DCT's performance in a multi-hop, multibottleneck environment, we use the topology shown in Figure 11(a). A total of 30 senders (where $S_{1}, S_{2}$, and $S_{3}$ represent a set of senders) and 11 receivers are used in this case. We generate long-lived flows, $S_{1} \rightarrow R_{1}, S_{2} \rightarrow R_{1}$ and $S_{3} \rightarrow R_{1}$. Note that $S_{1}$ competes with both $S_{2}$ and $S_{3}$ but at different links. Therefore, we expect the throughput of $S_{1}$ to be lower than $S_{2}$ and $S_{3}$ because the throughput of TCP flows is inversely proportional to the number of bottlenecks it traverses [15]. Figure 11(b) shows the average per-flow throughput in each sender set. Observe that with $\mathrm{L}^{2} \mathrm{DCT}, S_{1}$ achieves higher throughput compared to TCP and DCTCP and is also able to maintain better fairness.

\section{Co-existence with TCP}

TCP is a widely used congestion control protocol in cloud data centers [1], [7]. Therefore, we now evaluate $\mathrm{L}^{2}$ DCT's performance when it co-exists with TCP. Using a single bottleneck topology, we generate multiple long-lived flows and observe the effect of varying $k$ and $w_{c}$ on the relative throughput of $\mathrm{L}^{2} \mathrm{DCT}$ and TCP.

Figures 12(a) and 12(b) show the throughput as a function of $k$ for two cases: (a) one flow is generated by each protocol, and (b) each protocol generates two flows. Observe that as 


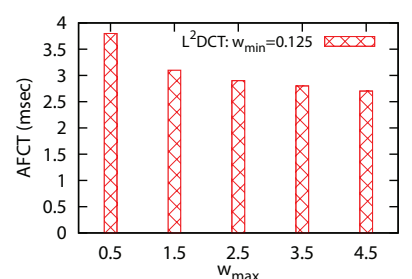

(a)

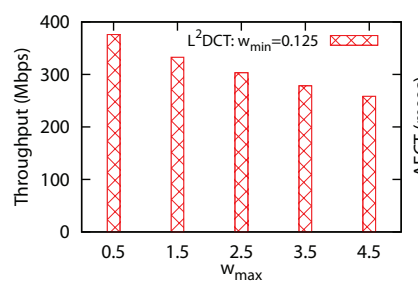

(b)

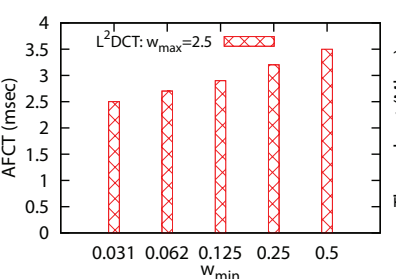

(c)

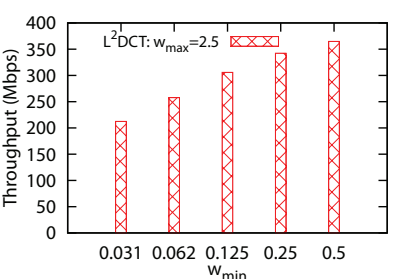

(d)

Fig. 9. Impact of varying $w_{\max }$ and $w_{\min }$ on the AFCT of short flows and the throughput performance of long-lived flows.

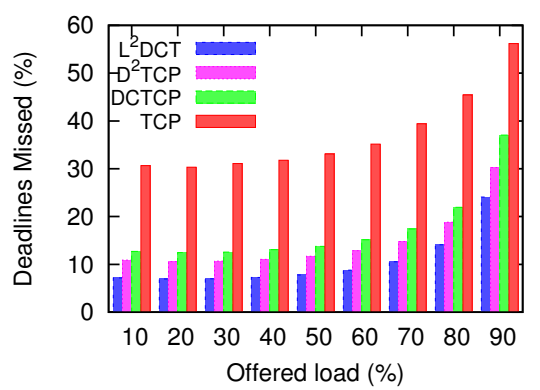

(a) Tight Deadlines

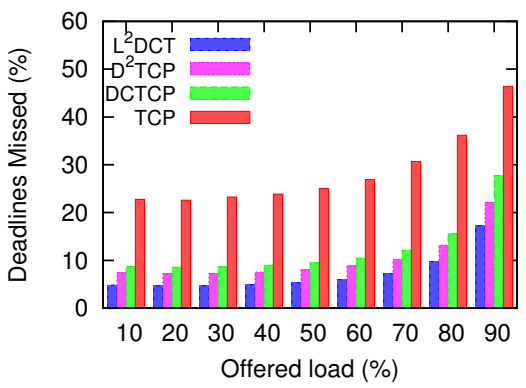

(b) Moderate Deadlines

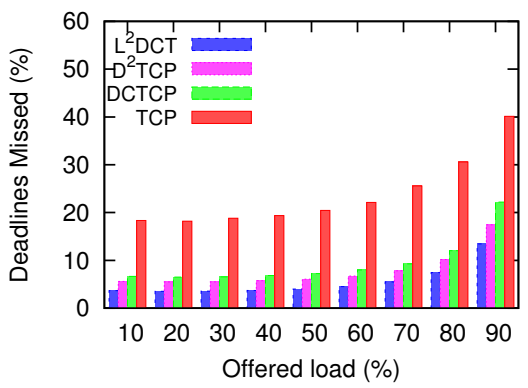

(c) Lax Deadlines

Fig. 10. Deadlines missed by various protocols. (a) Tight deadlines $(40 \mathrm{~ms})$. (b) Moderate deadlines $(60 \mathrm{~ms})$. (c) Lax ( $80 \mathrm{~ms})$. Observe that $\mathrm{L}^{2} \mathrm{DCT}$ misses the least number of deadlines.

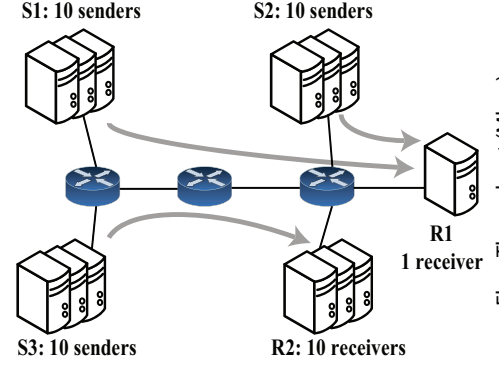

(a)

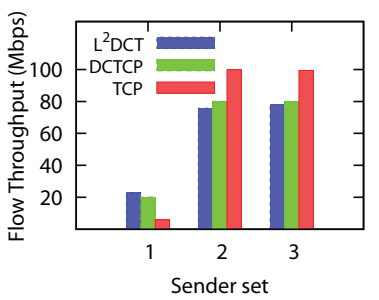

(b)
Fig. 11. Throughput performance under multiple bottleneck topology

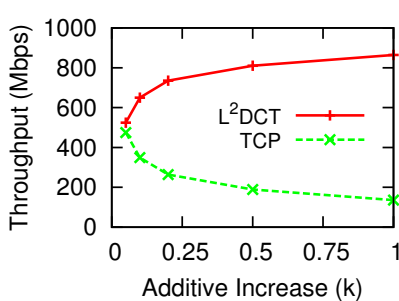

(a)

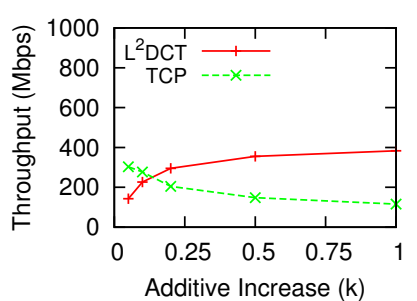

(b)
Fig. 12. Throughput of long-lived TCP and $\mathrm{L}^{2} \mathrm{DCT}$ flows when they co-exist. Each protocol generates (a) one flow and (b) two flows.

$k$ increases, $L^{2} D C T$ flows become more aggressive, thus achieving much higher throughput than TCP flows. Since there are very few concurrent long flows in data centers [1], using $k<0.25$ can provide suitable fairness between TCP and $\mathrm{L}^{2} \mathrm{DCT}$ flows. Note that $\mathrm{L}^{2} \mathrm{DCT}$ uses $k=0.05$ (as $\left.w_{\min }=0.125\right)$ for long flows.

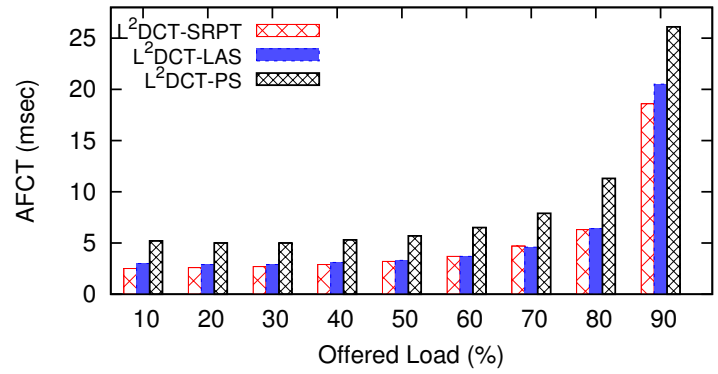

Fig. 13. AFCT under different scheduling disciplines

\section{E. Realizing Other Scheduling Policies}

In this section, we show how $\mathrm{L}^{2} \mathrm{DCT}$ can approximate a variety of scheduling policies such as SRPT and PS by adapting the flow weights. To realize SRPT, the weights are now adapted based on the remaining flow data instead of the data sent so far. For PS, we set $w_{c}=1$ for all flows. Figure 13 shows that LAS is a good approximation of SRPT. PS, however, results in a much larger AFCT.

\section{Real Testbed Implementation}

We built a small-scale testbed to evaluate the performance of $\mathrm{L}^{2}$ DCT in real network settings.

\section{A. Linux Implementation Details}

$\mathrm{L}^{2} \mathrm{DCT}$ requires very few changes at the end-hosts and none at the routers. It is implemented as a kernel module in Linux 2.6.38, which supports pluggable congestion control. $\mathrm{L}^{2} \mathrm{DCT}$ inherits important features of TCP such as retransmission and fast recovery mechanism. We used DCTCP's end host implementation for building $\mathrm{L}^{2} \mathrm{DCT}$.

a) Marking at the Switch: For realizing L $^{2}$ DCT's switch, we use the RED queue implementation in Linux. We set the 


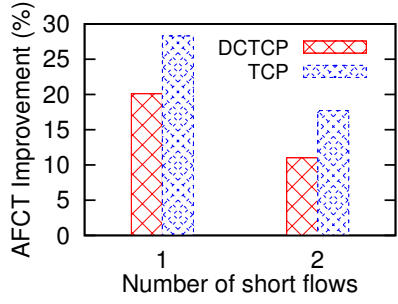

(a)

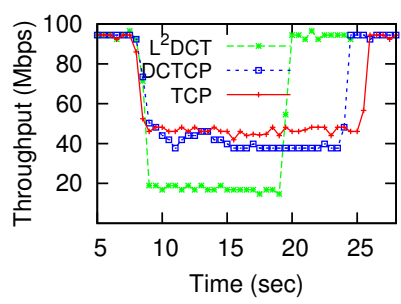

(b)
Fig. 14. Linux results for $\mathrm{L}^{2} \mathrm{DCT}$ (a) AFCT and (b) Long flow throughput.

low and high thresholds of RED queue to $K$ and perform marking based on the instantaneous rather than the average queue length as done in RED.

b) Sender-Side Modifications: $\mathrm{L}^{2} \mathrm{DCT}$ introduces changes in the additive increase and multiplicative decrease parameters used by TCP. In addition, it computes the fraction of marked packets to infer the degree of congestion at the bottleneck. $\mathrm{L}^{2} \mathrm{DCT}$ uses an array to hold pre-computed values of the backoff penalty $b$ for each $\alpha$ and weight $w_{c}$ in order to avoid floating point calculations.

c) Receiver ECN Echo Mechanism: L ${ }^{2}$ DCT introduces no changes at the receiver end and its ECN echo mechanism is the same as DCTCP.

\section{B. Linux Evaluation}

Our testbed comprises of three Linux machines, each equipped with a $100 \mathrm{Mbps}$ Realtek ethernet card. One machine acts as a client, one as a server and the other as a switch. We set $K$ at 8 packets. We compare $\mathrm{L}^{2}$ DCT's performance with DCTCP and TCP NewReno with ECN support.

We use Iperf for traffic generation. Due to the timer resolution limitations in Iperf, we generate and give maximum weight to flows of length $100 \mathrm{MB}$ and adapt the weight assignment policy accordingly. Note that this is just for the validation of our implementation and does not represent actual data center workloads.

We start a single long-lived flow in the background and generate multiple short flows simultaneously. We repeat each experiment 10 times for each number of flows. Figure 14(a) shows the improvement in AFCT of $\mathrm{L}^{2} \mathrm{DCT}$ over DCTCP and TCP as a function of the number of simultaneous short flows. Note that this represents a very high load scenario as each flow has $100 \mathrm{MB}$ of data to send. Observe that $\mathrm{L}^{2} \mathrm{DCT}$ improves the AFCT by up to $20 \%$ and $29 \%$ over DCTCP and TCP, respectively. Figure 14(b) shows the corresponding throughput of the long flow when one short flow arrives. Observe that with $\mathrm{L}^{2} \mathrm{DCT}$, the long flow throughput reduces to $\sim 20 \mathrm{Mbps}$ whereas the short flow gets $\sim 80 \mathrm{Mbps}$. This allows the short flow to finish quickly, thus causing the long flow to obtain maximum throughput sooner than other protocols. Since DCTCP and TCP are fair sharing protocols, short flows obtain $\sim 50 \mathrm{Mbps}$ of throughput with them. This causes the DCTCP and TCP short flows to finish $4 \mathrm{~s}$ and $5.5 \mathrm{~s}$ later, respectively.

\section{RELATED WORK}

The relevant literature on congestion control, scheduling, and reducing latency is vast. Therefore, in this section, we only summarize some of the most relevant works.

CUBIC [16], BMCC [17], XCP [18], and delay-based congestion control protocols, such as FAST [19] and CTCP [20], all successfully improve performance in high BDP networks. However, these protocols approximate fair sharing and thus are sub-optimal in terms of completion times. Rate Control Protocol (RCP) improves the AFCT by reducing the startup latency of flows [8]. However, RCP is also a fair sharing protocol and requires router hardware modifications [1].

In [21], authors propose HULL which is based on capping utilization at less than link capacity. By sacrificing some amount (e.g., 10\%) of bandwidth, HULL can reduce the average and tail latencies. Our work is complementary to HULL. In particular, $\mathrm{L}^{2} \mathrm{DCT}$ can be combined with their proposal to further reduce completion times. However, it is useful to note that $\mathrm{L}^{2} \mathrm{DCT}$ reduces completion times without sacrificing link capacity. In [22], Zats et al. propose DeTail, an in-network congestion management mechanism that reduces the flow completion time tail in data center environments. However, DeTail does not target AFCT. Unlike DeTail, ${ }^{2}$ DCT can save up to $\sim 45 \%$ over DCTCP and $\sim 95 \%$ over TCP. In addition, it reduces the completion time of almost every flow.

Earliest Deadline First (EDF) is provably optimal when individual packets are associated with deadlines. However, when associated with flows, applying EDF to individual packets is not only suboptimal but can increase network congestion [4].

QCN, an optional standard for Ethernet, uses multibit feedback from the switches to reduce recovery time during congestion. However, QCN cannot span beyond L2 domain limiting its scope of application [1].

To approximate LAS, one could use priority queuing at the switches. However, prior studies show that using twolevel priorities, TCP/RCP with priority queuing suffer from high loss rate and falls behind $\mathrm{D}^{3}$ [4]. Further, increasing the priority does not significantly improve performance as flows within each class may have widely different sizes and yet they are not differentiated. Consequently, large number of priority classes are needed, however, switches nowadays provide only a small number of classes, usually no more than ten [4], [10].

Yang et al. [23] proposed TCP SAReno, which adapts AIMD parameters based on the residual flow size assuming droptail queuing. First, it uses a small number of classes and therefore, faces the same issues as TCP with priority queuing. Second, it uses fixed parameters for each class, and thus considerably degrades the performance of long flows even in the absence of short flows. Zieglar et. al. [24] also proposed to dynamically adapt AIMD parameters for improving the startup latency of short flows. However, with their protocol, a short flow achieves no higher throughput than long flows for the protocol to be incentive-compatible with TCP.

Several recent works, such as [25], show the benefit of using multi-path TCP, ranging from improved network utilization to better reliability. However, developing a multi-path version of $\mathrm{L}^{2} \mathrm{DCT}$ is part of future work. 


\section{DisCUSSION AND FUTURE WORK}

Fairness. One may argue that the performance gains of $\mathrm{L}^{2} \mathrm{DCT}$ over other protocols are due to the fact that it unfairly penalizes long flows. It turns out that the performance improvement over fair sharing protocols does not usually come at the expense of long jobs. Bansal et al. [9] showed that with SRPT, at least $99 \%$ of the jobs have smaller completion times compared with fair sharing ${ }^{3}$. Moreover, this percentage increases even further when the traffic load is less than half. Typical data center workloads are generally less than 50\% [6]. Further, if desired, an operator can always set the weight of flows to achieve a wide range of bandwidth sharing criteria, including fairness. For instance, to achieve fair sharing, an operator could set $w_{c}=1$ for all flows.

Gaming the System. One could ask whether users will have an incentive to improve the completion time of their flows by splitting them into smaller flows. While the incentive is greater in case of $\mathrm{L}^{2} \mathrm{DCT}$ compared to fair sharing protocols, similar issue also arises in TCP and RCP, where users may achieve higher aggregate throughput by splitting a flow into smaller ones, as well as in $\mathrm{D}^{3}$, where users may request a higher rate than the flow actually needs. With PDQ, however, the incentive may be even greater as PDQ does preemption of flows whereas $\mathrm{L}^{2} \mathrm{DCT}$ does not. To address this, users with multiple flows can be penalized by changing their weights. However, designing a scheme to achieve this end remains a future work. We would like to point out that in data center environments, connectivity to the external Internet is typically managed through application proxies that effectively separate internal traffic from external, therefore, issues of fairness with conventional TCP outside are irrelevant [1].

When flow completion time is not the priority. For real-time applications such as VoIP, flow completion time is not the best metric. For such applications, a constant $w_{c}$ can be assigned, making it equivalent to a DCTCP flow.

Stability. If all flows are short and thus demand a high share of the bandwidth, network overload may occur. However, $\mathrm{L}^{2}$ DCT's backoff mechanism guards against such overload on two fronts: (1) When $\alpha$ approaches one, $\mathrm{L}^{2} \mathrm{DCT}$ defaults to the same backoff as TCP, therefore, $\mathrm{L}^{2}$ DCT's worst case stability is similar to that of TCP. (2) We limit the maximum value of $w_{c}$ to 2.5, which limits the aggressiveness of short flows.

\section{CONCLUSION}

We design and implement $\mathrm{L}^{2} \mathrm{DCT}$, a data center transport protocol, which targets minimizing the flow completion times by approximating the LAS scheduling discipline. $\mathrm{L}^{2}$ DCT can co-exist with TCP and delivers high application throughput by meeting more deadlines than existing protocols. For a wide range of data center workload scenarios $\mathrm{L}^{2} \mathrm{DCT}$ provides up to $45 \%$ and $95 \%$ reduction in AFCT over DCTCP and TCP. The $99^{t h}$ percentile improvement in flow completion times over DCTCP is up to $37 \%$ and $53 \%$ when the flow size distribution is uniform and Pareto, respectively. Though $\mathrm{L}^{2} \mathrm{DCT}$ is deadline unaware, owing to its goal of minimizing

\footnotetext{
${ }^{3}$ Assuming M/G/1/SRPT queueing model with heavy-tailed distributions.
}

flow completion times, it would miss up to about $35 \%$ fewer deadline compared to a recent deadline-aware protocol $\mathrm{D}^{2} \mathrm{TCP}$ for tight as well as lax deadlines. These improvements come at a cost of small throughput degradation for background flows; although this does not impact the end-user response time, it can still be easily addressed by adjusting $w_{c}$. However, data center operators must evaluate the impact of using $\mathrm{L}^{2} \mathrm{DCT}$ using their own traffic and network profile. We also implemented $\mathrm{L}^{2} \mathrm{DCT}$ on Linux demonstrating that it does not require any flow specific information from applications and can be easily deployed without requiring any additional hardware and software support.

\section{REFERENCES}

[1] M. Alizadeh, A. Greenberg, D. Maltz, J. Padhye, P. Patel, B. Prabhakar, S. Sengupta, and M. Sridharan, "Data center tcp (dctcp)," in SIGCOMM'10.

[2] T. Hoff, "Latency is everywhere and it costs you sales how to crush it," July 2009, http://highscalability.com/blog/2009/7/25/latencyis-everywhere-and-it-costs-you-sales-how-to-crush-it.html.

[3] D. Abts and B. Felderman, "A guided tour of data-center networking," Commun. ACM, vol. 55, no. 6, pp. 44-51, June 2012.

[4] C. Wilson, H. Ballani, T. Karagiannis, and A. Rowstron, "Better never than late: Meeting deadlines in datacenter networks," in SIGCOMM'11.

[5] T. Benson, A. Akella, and D. Maltz, "Network traffic characteristics of data centers in the wild," in IMC' 10 .

[6] B. Vamanan, J. Hasan, and T. N. Vijaykumar, "Deadline-aware datacenter tcp (d2tcp),' in SIGCOMM'12.

[7] H. Wu, Z. Feng, C. Guo, and Y. Zhang, "Ictcp: Incast congestion control for tcp in data center networks," in Co-Next'10.

[8] N. Dukkipati, M. Kobayashi, R. Zhang-Shen, and N. McKeown, "Processor sharing flows in the internet," in IWQoS, 2005.

[9] N. Bansal and M. Harchol-Balter, "Analysis of srpt scheduling: investigating unfairness," in SIGMETRICS'01.

[10] C.-Y. Hong, M. Caesar, and P. B. Godfrey, "Finishing flows quickly with preemptive scheduling," in SIGCOMM'12.

[11] I. A. Rai, G. Urvoy-Keller, and E. W. Biersack, "Analysis of las scheduling for job size distributions with high variance," in SIGMETRICS'03.

[12] K. K. Ramakrishnan and S. Floyd, "The addition of explicit congestion notification (ECN) to IP," in IETF RFC 3168, Sep 2001.

[13] G. Appenzeller, I. Keslassy, and N. McKeown, "Sizing router buffers," in SIGCOMM'04.

[14] V. Vasudevan, A. Phanishayee, H. Shah, E. Krevat, D. Andersen, G. Ganger, G. Gibson, and B. Mueller, "Safe and effective fine-grained tcp retransmissions for datacenter communication," in SIGCOMM'09.

[15] Y. Xia, L. Subramanian, I. Stoica, and S. Kalyanaraman, "One more bit is enough," in SIGCOMM'05.

[16] I. Rhee and L. Xu, "CUBIC: A new TCP-friendly high-speed TCP variant," in PFLDNet'05, 2005.

[17] I. A. Qazi, L. L. H. Andrew, and T. Znati, "Congestion control with multipacket feedback," in IEEE/ACM Trans. on Networking, 2012.

[18] D. Katabi, M. Handley, and C. Rohrs, "Internet congestion control for high bandwidth-delay product networks," in SIGCOMM'01.

[19] C. Jin, D. Wei, and S. Low, "FAST TCP: Motivation, architecture, algorithms and performance," in INFOCOM'04.

[20] K. Tan, J. Song, Q. Zhang, and M. Sridharan, "A compound TCP approach for high-speed and long distance networks," in INFOCOM'06.

[21] M. Alizadeh, A. Kabbani, T. Edsall, B. Prabhakar, A. Vahdat, and M. Yasuda, "Less is more: Trading a little bandwidth for ultra-low latency in the data center," in NSDI'12.

[22] D. Zats, T. Das, P. Mohan, D. Borthakur, and R. Katz, "DeTail: Reducing the Flow Completion Time Tail in Datacenter Networks," in SIGCOMM'12.

[23] S. Yang and G. De Veciana, "Enhancing both network and user performance for networks supporting best effort traffic," IEEE/ACM Trans. on Networking, 2004.

[24] T. Ziegler, H. Tran, and E. Hasenleithner, "Improving perceived web performance by size based congestion control," NETWORKING'04.

[25] C. Raiciu, S. Barre, C. Pluntke, A. Greenhalgh, D. Wischik, and M. Handley, "Improving datacenter performance and robustness with multipath tcp," in SIGCOMM'03. 